\title{
Electromagnetic fault injection against a complex CPU, toward new micro-architectural fault models
}

\author{
Thomas Trouchkine - Sébanjila Kevin Bukasa - Mathieu Escouteloup • \\ Ronan Lashermes • Guillaume Bouffard
}

Received: date / Accepted: date

\begin{abstract}
The last years have seen the emergence of fault attacks targeting modern central processing units (CPUs). These attacks are analyzed at a very high abstraction level and, due to the modern CPUs complexity, the underlying fault effect is usually unknown. Recently, a few articles have focused on characterizing faults on modern CPUs.

In this article, we focus on the electromagnetic fault injection (EMFI) characterization on a bare-metal implementation. With this approach, we discover and understand new effects on micro-architectural subsystems. We target the BCM2837 where we successfully demonstrate persistent faults on L1 instruction cache, L1 data cache and L2 cache. We also show that faults can corrupt the memory management unit (MMU). To validate our fault model, we realize a persistent fault analysis to retrieve an AES key.
\end{abstract}

Keywords Physical attacks · Fault models · Electromagnetic Fault Injection (EMFI) - System-on-Chip (SoC)

S. K. Bukasa · M. Escouteloup

INRIA/CIDRE, Rennes, France

R. Lashermes

INRIA/SED, Rennes, France

T. Trouchkine · G. Bouffard

National Cybersecurity Agency of France (ANSSI)

51, boulevard de La Tour-Maubourg

75700 Paris 07 SP, France

E-mail: thomas.trouchkine@ssi.gouv.fr

G. Bouffard

DIENS, École normale supérieure,

CNRS, PSL University, Paris, France

E-mail: guillaume.bouffard@ens.fr

\section{Introduction}

Sensitive operations are usually performed on secureoriented devices such as smartcards. These kinds of devices are hardened microcontroller units (MCUs) designed to be tamper resistant against hardware and software attacks. They are evaluated under a scheme, e.g. Common Criteria, to ensure a high security level. Smartcards are the most studied secure-oriented devices.

Recently, sensitive operations are increasingly performed on more powerful and complex devices: the systemon-chips (SoCs). These SoCs are powered by central processing units (CPUs) with a complex micro-architecture including memory virtualization, several cache levels, speculative or out-of-order execution.

To behave correctly, components have to fulfill physical constraints. These constraints are directly linked to their design and, if they are respected in their nominal operation point, it is possible to break these constraints conditions by inducing stimuli in the component as presented in Figure 1.

When a stimuli perturbs the component, it is pushed to its operation limit. Therefore, faults can be induced.

Among all the fault injection methods, electromagnetic fault injection (EMFI) has been demonstrated to be effective on the complex CPUs with a minimal effort on target preparation [8] and without chip alteration.

Complex CPUs are tuned for the best performance/energy consumption trade-off and are focused on software hardening. However, hardware security is not the main 


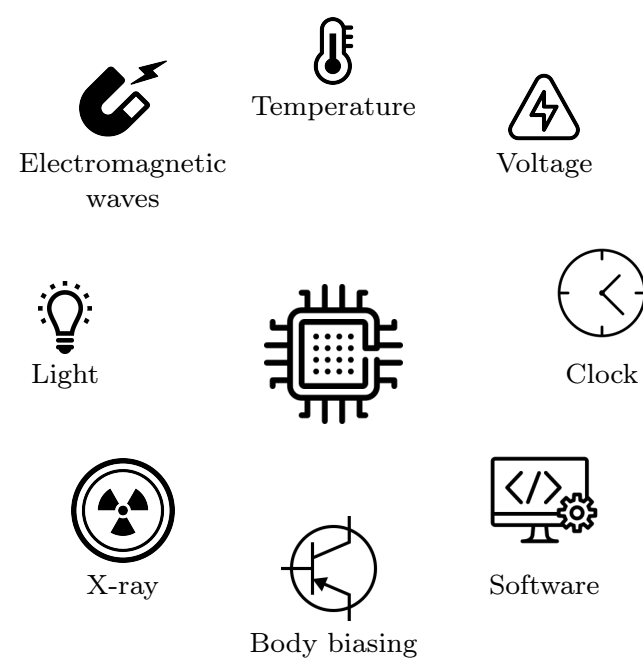

Fig. 1: Stimuli able to perturb a component

concern and past works [8, 11, 12, 18, 19, 20, 25] have shown that physical attacks are effective against them.

These articles demonstrate the need for hardening complex CPUs against fault attacks. To achieve that goal, we first need to understand the effects of such faults on the micro-architecture.

To model the fault effects, literature $[1,2,10,13,15$, $26,28]$ covers mostly a simple component: the MCU. The MCU is cheaper and smaller than a complex CPU without advanced micro-architectural components. Their works improve the hardware security of MCU and a lot of introduced countermeasures are embedded in smartcards. However, the obtained fault model cannot be scaled up on complex CPUs embedded in SoCs due to the latter's hardware complexity.

Some recent papers $[14,21]$ have proposed fault characterizations on complex CPUs. However, since they use a device running a complex operating system (OS), their analysis cannot precisely infer the impact on microarchitectural elements.

In this work, we propose instead to run our opensource characterization software ${ }^{1}$ as a bare-metal application for the Raspberry Pi 3 B (RPi3)'s CPU embedded in the BCM2837 SoC. This implementation allows us to have control of software/hardware interactions. Therefore, we can realize a finer grained characterization on several micro-architectural components. To fault the BCM2837 CPU, we first make EMFI and we next use a forensic process based on JTAG debug port

\footnotetext{
1 This implementation and the experiment data are released as open-source software (MIT License) here: https://gitlab. inria.fr/rlasherm/rpi3_fault_analysis.
}

to understand fault effects.

In this article, we highlight several new fault effects at the micro-architectural level, in particular in the memory hierarchy.

This article is organized as follows. The BCM2837 is described in section 2. On its complex CPU, we run a nested for loop and we vary the injection delay. The experimentation modus operandi is explained in section 3 .

Regarding this experimentation, we observed faults on different micro-architecture blocks:

- on the L1 instruction cache where we achieve a persistent fault in the L1 instruction cache as described in section 4 ,

- on the memory management unit (MMU); we confuse the virtual-to physical memory mapping. This fault is introduced in section 5,

- on the L2 cache; we also achieve a persistent fault in the L2 cache demonstrated in section 6 .

A fault exploitation demonstration is given in section 7 with a persistent fault in the L1 data cache, where an AES cryptographic key is recovered with one fault injection and $2^{24}$ hypotheses.

We finish with propositions to protect complex CPUs against these attacks in section 8 . Section 9 puts our work in the state of the art and we conclude in section 10 .

\section{BCM2837 description}

The RPi3 is a low-cost single-board computer. It features a complete system able to run a complex OS such as Linux or Windows and their applications. The SoC powering this board is the BCM2837 from Broadcom, a $28 \mathrm{~nm}$ quad-core Cortex-A53 CPU running at $1.2 \mathrm{GHz}$ with the help of a dual-core VideoCore IV GPU at $400 \mathrm{MHz}$. This SoC also features $512 \mathrm{kB}$ of cache memory and various wireless or contact connections such as HDMI, Wi-Fi, ethernet, USB, etc.

Our experiments are performed with our own software stack, with only one core active. Indeed, even if we tested EMFI with several cores enabled, it is hard to pinpoint the exact effect on the micro-architecture in this configuration since we cannot easily link a fault in unified memory with a particular core. Therefore, to increase our confidence in our characterization, we enable only one core.

To control the chip's behavior, we have implemented the minimum to run our applications: initialization of 
JTAG, UART, GPIO, CPU caches and MMU. We want to stress out that no complex OS is running during our experiments in sections $4,5,6$ and 7 , to avoid interference that could hinder our ability to infer more precise fault models. In particular, we try to avoid the context switching effects due to preemptive scheduling by the OS, the error recovery mechanisms (if an error occurs, we want to know it) and the cache maintenance performed by the OS.

In order to later explain the causes of the failures observed, we describe in more details two important micro-architecture blocks of this complex CPU: the cache hierarchy and the MMU.

\subsection{Cache hierarchy}

In modern systems, memory accesses are a lot slower than the arithmetic logic unit (ALU). To avoid losing too much performance to this latency difference, small and fast memories called caches are used to mirror a part of the memory space.

First, in the targeted CPU, each core has two L1 caches (the smallest and fastest kind), one dedicated to instructions (L1I), one to data (L1D). These caches are $16 \mathrm{kB}$ in size with $64 \mathrm{~B}$ line width.

Second, the second layer of cache memory, the L2 cache, is common to all cores and thus provides a unified view of the memory space. Its size is $512 \mathrm{kB}$ with $64 \mathrm{~B}$ line width.

The cache organization is displayed in Figure 2. The bus sizes between memory elements are given in Table 1

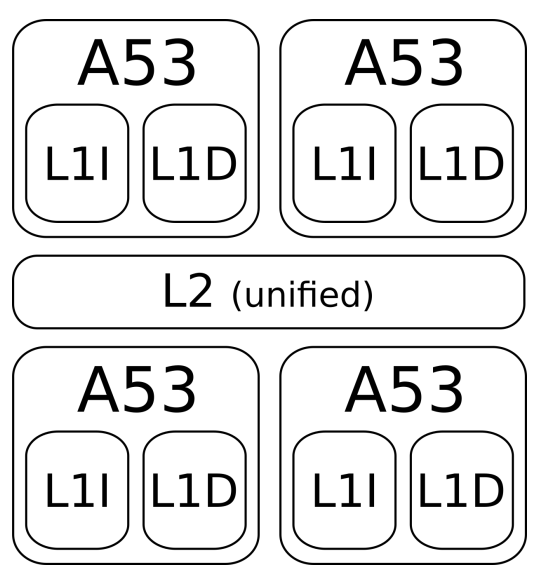

Fig. 2: BCM2837 memory hierarchy.

\begin{tabular}{|l|l|l|}
\hline Source & Destination & Bus size (bits) \\
\hline RAM & L2 & 128 \\
L2 & L1I & 128 \\
L2 & L1D & 128 \\
L1D & L2 & 256 \\
L1D & pipeline & 64 \\
pipeline & L1D & 128 \\
\hline
\end{tabular}

Table 1: BCM2837 memory hierarchy bus sizes

\subsection{Memory management unit}

The MMU is a central component for all multi-application systems. It aims to virtualize the physical memory of the system into a virtual one to segregate each app in their own address space. Therefore, the CPU only works with virtual addresses, and during a memory access to one of them, the MMU translates it into the corresponding physical address, which is transmitted to the memory controller. The information required for the translation of an address is called a page table entry (PTE), and it is stored in the physical memory and cached in the translation lookaside buffer (TLB). There is a PTE for all allocated pages in the physical memory. Our bare-metal implementation allocates the whole address space with an identity mapping (virtual and physical addresses are the same) with $64 \mathrm{kB}$ pages.

In modern systems, the translation phase does not only compute the physical address but also realizes different checks. These checks are monitoring if the page can be written or not, which kind of process (user or supervisor) can access it or should the page be stored in cache or not.

Among all its roles, the MMU is also a security mechanism. Ensuring that a read-only page cannot be written to and ensuring that only authorized processes can access their corresponding pages. This last security mechanism is the memory partitioning. On multiapplication systems, it avoids a process to spy or corrupt the memory area used by another one.

In complex OSs, the MMU and the PTEs are critical assets set up by the kernel.

\section{Experimental process methodology}

In this section, we introduce our benchmark to characterize the fault model and which micro-architecture blocks are faulted.

\subsection{Electromagnetic fault injection bench}

Some apparatus are required in order to inject faults into the BCM2837. Our experimental setup has been 
designed to be highly configurable and to operate at higher frequencies than most setups targeting microcontrollers. First, we use a Keysight 33509B (waveform generator) to control the delay between a trigger issued by the RPi3 board before the instructions of interest. Second, a Keysight 81160A generates the signal shape for the EM injection: one sinus period. A sinus is used instead of the usual pulse since it gives fewer harmonics at the output of the signal generation chain. Third, this signal is amplified with a Milmega 80RF1000-175 $(80 \mathrm{MHz}-1 \mathrm{GHz}$ bandwidth). Finally, the high-power signal is connected to a Langer RF U 5-2 near-field probe, in contact with the packaged chip. A part of this electromagnetic energy is, therefore, transmitted in the chip's metal lines, which can lead to a fault.

The minimum latency between the initial trigger and the faulting signal reaching the target is high: around $700 \mathrm{~ns}$. As a consequence, the targeted application must run enough time to be reachable by our fault injection bench.

\subsubsection{Bench parameters}

To properly inject a fault, the experimenter must tune several parameters, namely the 1) injection signal, 2) the probe spatial location, and 3) temporal synchronization.

Fault effects are reproducible with a low ratio; meaning that if a fault has been achieved, it will be achieved again with the same parameters but only for a small ratio of the fault injections. In other cases, no failure occurs, or another effect is observed (mostly due to jitter). Unfortunately, we are not able to measure the fault ratio. Indeed a manual forensic analysis must be performed in order to establish the cause for a fault. Since this step is not automated, it is too time consuming to measure a fault ratio.

Injection signal. The signal parameters (shape, frequency, number of periods) have an optimal value with respect to our requirements:

- The shape is a sinus to reduce the presence of harmonics in the amplification chain.

- The frequency (275 MHz) maximizes the probe gain as measured in the probe characterization phase. As a consequence the sinus period is $3.6 \mathrm{~ns}$, or $\approx 4.3$ clock periods.

- The amplitude $(-14 \mathrm{dBm})$ has been manually tuned during the fault injection until the desired effect is achieved: if too low, no faults are observed; if too high, the chip crashes and must be rebooted.
Probe spatial location. A sensitivity map has been made to find the sensitive places on the chip. By setting a high signal amplitude, we repeatedly try to inject a fault in every possible place on the chip. Next, we observe where the faults (the tested application, Listing 1, returns an incorrect result or no result at all) are obtained. The result, shown in Figure 3, clearly indicates an area of interest.

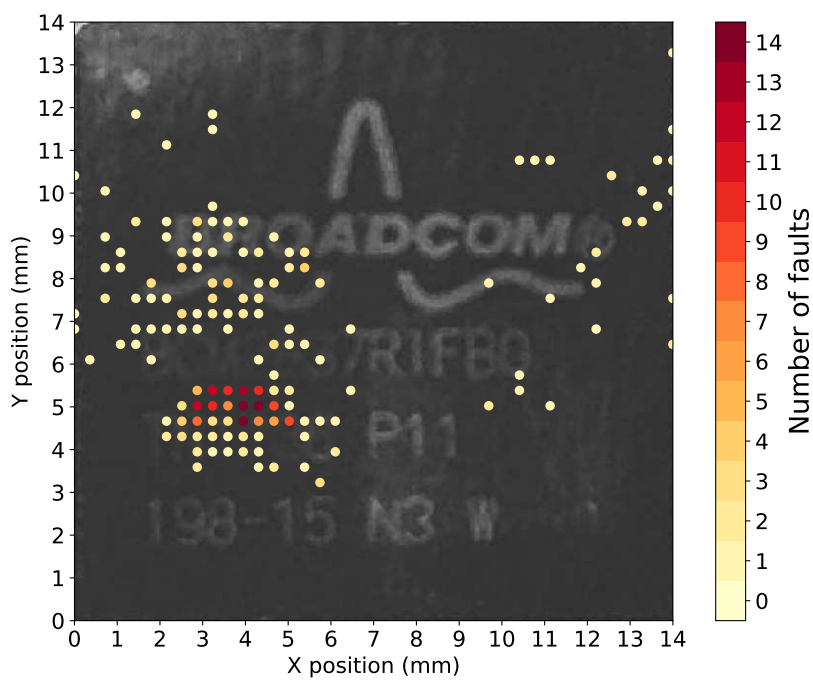

Fig. 3: EMFI BCM2837 sensitivity map

(Dot size is not correlated with probe size.)

For all experiments below, the probe is placed at the most sensitive location $(X=4 \mathrm{~mm}, Y=4.5 \mathrm{~mm}$ on Figure 3).

Temporal synchronization. The main difficulty for fault injection is the temporal synchronization: when to inject a fault on the targeted and vulnerable instructions.

We first need a temporal reference, given here by a GPIO: a rising electrical edge is sent to a board pin by our application just before the area of interest. This signal is used by our apparatus to trigger the fault injection.

Our setup is using the evaluator approach: the attacker can instrument the system to ease the experiments. In the case of a real attack, the adversary would have to generate this trigger signal: it can be done by monitoring communications, IOs, or EM radiation to detect patterns of interest. In all cases, it is a tricky business, highly application dependent.

The trigger signal is just part of the problem: from that moment on, we must wait the correct duration to inject the fault. In other words, we want to control the injection time relatively to the trigger signal, in order to inject a fault when processing the targeted 
instruction. Propagation times are not negligible. On a complex CPU, the issue is more difficult due to the memory hierarchy. Since cache misses are highly unpredictable, they imply a corresponding jitter. It is difficult to predict the duration of memory access accurately and therefore, the waiting time to inject the fault.

Synchronization is a problem, but not an obstacle at this point. Indeed, the attacker only has to inject faults until the correct effect is achieved. Because of the jitter, for the same delay (time expected between the trigger and fault injection), different timings are tested with respect to the running program. For the same bench timing parameters, we will inject a fault during a different instruction at each execution, but a different one among a small set of instructions, depending on the jitter. If a fault with an interesting effect is possible, it is eventually achieved.

Additionally, as we will see in the next sections, memory transfers are particularly vulnerable to EMFI. They are also slower than the core pipeline, allowing for a bigger fault injection timing window.

\subsection{Forensic methodology}

Contrary to the state-of-the-art fault model characterization on complex CPUs, we target a bare-metal system, meaning that no complex OS, to characterize the effect of a fault in the micro-architecture. Removing the complex OS aims at improving the fault effect characterization with a better granularity by accessing to low-level micro-architecture blocks and instrumenting them.

As the targeted component is closed, we do not have any insider information and no way to analyse the hardware layout. Therefore, we can only characterize a fault from the instruction set architecture (ISA). Moreover, we have also limited means to explore what is happening in the system, mainly the JTAG. With it, we are able to halt the chip execution to read or write the register values and to read and write memory as seen by a particular core (with a data viewpoint).

Therefore, to pinpoint the particular effects of a fault injection, we force the system state such that an observable change gives us information on the fault mechanism. In particular, we have put several cache invalidation instructions in memory. During our forensic process, we can therefore call them by modifying the program counter (PC) by writing directly to it with the JTAG.

A technique used to detect the fault location is to execute the suspected instructions step-by-step (with the JTAG), and the verify the side effects after each instruction (by reading memory and registers).
Our software footprint has to be minimal to maintain controllability on the system state. As such, we will not describe how to breach a particular system with our faults since any exploit is highly applicationdependent, and our setup is not representative of a standard application environment. Instead, we will suggest exploit strategies: how a malicious attacker could use such faults?

A demonstration of a fault exploitation, given in section 7, is done on our own AES encryption implementation. However, those results cannot be extrapolated to any real-world application.

\subsection{Characterization modus operandi}

The application targeted during this experiment is two nested loops shown on Listing 1. This program is built without compiler optimizations. We set the compiler optimization level at -O0 to have better debuggable code and correspondence between C-code and generated assembly. Moreover, -O0 increases the number of memory accesses. This behavior helps us to analyse them against fault attacks.

Listing 1: Loop target application

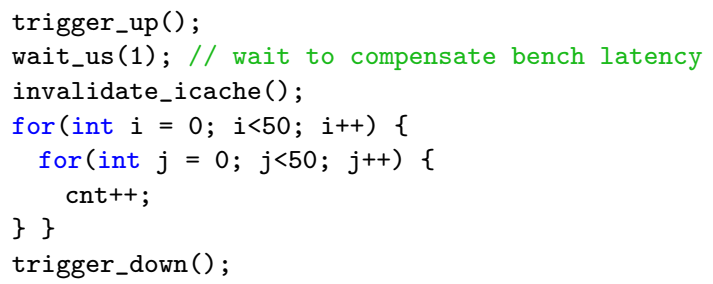

Before running the nested for loop, we invalidate the CPU caches (line 3) to detect modifications. Our bench has a latency of $700 \mathrm{~ns}$, so we can still hit this memory transfer. To be able to observe the effect of a fault on the full-timing range, a $1 \mu$ s (line 2) wait has been inserted between the trigger and the cache invalidation. Moreover, a MMU is enabled to be closer to market deployed applications.

A fault occurs when the cnt variable value at the end of the program is not equal to 2500 or if the program crash. When a fault is detected, we use the JTAG to re-execute our for loop, in the Listing 1, by directly setting the PC value at the start of the loops. Executing instruction by instruction, we monitor the expected side effects. This execution is done without fault injection.

Based on this code, we vary the injection delay. Varying the injection delay confuses different microarchitecture blocks: L1 and L2 caches and the MMU. The L1 and L2 caches are confused at 3134 ns and the MMU is targeted at $25400 \mathrm{~ns}$. 
With this setup, we disclose different fault models with EM fault injection on L1I cache, on MMU and on L2 cache.

\section{Fault on the instruction cache}

In this experiment, we achieve a fault in the L1 instruction cache (L1I).

4.1 On the impossibility to fault the instruction execution flow

Before reporting our positive results, we must report a negative one. Contrary to previous works on microcontrollers (where, e.g. instructions are replaced with a NOP instruction), we are not able to prevent or modify the execution of an instruction directly. Even if we cannot be sure that no set of experimental parameters would achieve such a fault, we thoroughly explored the parameters without success.

Instead, we get faults during cache transfers, which is probably because the buses involved in these transfers are easily coupled with our EM probe. In the case of a minimal application that can fit in the cache, the memories are filled only once at the first execution. As a consequence, to repeatedly observe our fault, we invalidate the cache memory of interest (L1I) just before launching our application, artificially triggering a memory transfer.

\subsection{Forensic}

As the instruction cache is invalidated before the loop of the Listing 1 is executed, the following instructions must be (re)loaded into the cache before execution. Moreover, it is this memory transfer that we target with our fault injection. By executing the same application with and without the cache invalidation and measuring the duration of the high state of the trigger, we deduce that loading instructions in the cache has an overhead of $2 \mu \mathrm{s}$.

To analyze the fault, we debug, without fault and step by step, the for loop through the JTAG interface. Each instruction was correctly executed except for the add instruction, in red, at address $0 \times 48$ a08 on Listing 2.

Listing 2: Assembly version of the for loop (Listing 1) without optimisation.

0x48a04: b94017a0 ldr x0, [x29,\#20]
0x48a08: 11000400 add x0, x0, \#0x1
0x48a0c: b90017a0 str x0, [x29,\#20]

0x48a10: b9401ba0 ldr x0, [x29,\#24]

0x48a14: 11000400 add $\mathrm{x} 0, \mathrm{x} 0, \# 0 \mathrm{x} 1$

0x48a18: b9001ba0 str x0, [x29,\#24]

0x48a1c: b9401ba0 ldr x0, [x29,\#24]

0x48a20: 7100c41f cmp x0, \#0x31

0x48a24: 54ffff0d b.le $48 \mathrm{a} 04<10 o p+0 x 48>$

...

By monitoring the $\mathrm{x} 0$ register before and after the cnt incrementing instruction, in red in the Listing 2, we observe that the value is kept unchanged: the increment is not executed. Since the fault is still present after the EM injection, we can conclude that a wrong instruction value is stored in L1I. We confirm this fault model by executing an L1I cache invalidation instruction ic iallu $u^{2}$. By re-executing our application, the fault has disappeared.

We can infer that the injected fault has affected a part of the L1I cache. However, it is impossible to access (read) the new instruction value. Since the fault happens during the cache filling, we can suppose that the memory transfer had been altered.

Moreover, this fault model matches the observation made by Trouchkine et al. in [21]. In this work, the authors characterize a fault model while perturbing a BCM2837 using EMFI. The model they observe is the corruption of the executed instruction second operand, which is forced to 0 . This ISA fault model matches the one we observe in the cache as the corruption of the instruction add $\mathrm{x} 0, \mathrm{x} 0, \# 0 \mathrm{x} 1$ by forcing the second operand to 0 gives the instruction add $\mathrm{x} 0, \mathrm{x} 0, \# 0 \mathrm{x} 0$ which explains why the increment is not executed as we add 0 to the register. They identify that their faults happen during the instruction fetch. However, as they work with an OS, they cannot reliably test if the fault they observe is persistent in the cache, while we can with our bare-metal setup.

\subsection{Exploits}

This fault is one of the easiest to exploit since it induces an instruction replacement fault model, where an instruction is replaced by another. It can often be reduced to the classical instruction skip model, if the new instruction has no side effects on the application. Therefore, most exploits based on this classical model apply here. Since the faulted value is still present in the cache, it will stay faulted until the cache is invalidated: we can call this model "sticky instruction skip". Bukasa et al. [7] demonstrated applications of this fault model: hijacking the control flow and initiating a return-oriented programing (ROP) attack, among others.

2 We set the PC value to the ic iallu instruction address in memory. 


\section{Targeting the memory management unit}

The MMU is a critical component of the complex CPUs. It is in charge of the virtual memory mechanism and ensure memory partitioning between applications. In this section, the fault corrupts the virtual-to-physical memory mapping albeit in an uncontrolled manner. The targeted application is the same as in section 4, shown in Listing 1; only the fault injection timing has changed. We inject the fault at $25400 \mathrm{~ns}$ after the trigger instead of $3144 \mathrm{~ns}$ when the L1I is confused. The faults, analyzed in this section, always crash the program in Listing 1 .

We show in this section that this delay enables to perturb the MMU micro-architectural block. Regarding the results from the other characterization works in this paper, the cache hierarchy is the most sensitive CPU micro-architectural block.

\subsection{Configuration of a working MMU}

To understand the fault effect on the MMU, we begin to explore the state of an application without any fault. This state is a legitimate one.

\subsubsection{Page tables}

The page tables are used to memorize the mapping between virtual and physical memory. In our configuration, we have 3-page table directory (PTD) (mapping $512 \mathrm{MB}$ chunks), and for each of them, we have 8192 PTE pages of $64 \mathrm{kB}$.

In the page tables, the most and least significant bits are used for the page metadata (access rights, caches configuration, etc.).

\subsubsection{Translation lookaside buffer}

TLBs (plural since there are several of them) are small buffers used to speed up virtual-to-physical memory translation. As in a cache memory, the last mappings are saved from being reused later without a full-page walk by the MMU. In the targeted CPU, TLB hierarchy mirrors cache hierarchy: the TLB designates the unified Level2 buffer while micro-TLBs are dedicated to instructions or data in each core.

\subsubsection{Operating system}

In our bare-metal application, all the pages are initialized in the page tables with an identity mapping (virtual and physical addresses are identical). In a system with an OS, pages are allocated on-the-fly. On the one hand, this simplifies the forensic analysis since we are sure that page tables are correct prior to the fault. On the other hand, interesting faults may be missed if the OS page allocation is disrupted.

\subsection{Forensic}

To reconstruct the memory mapping, we use a pair of instructions computing the physical address (and the corresponding metadata) for a given virtual one. A script has been designed to extract the memory mapping. By using the JTAG, first, the two instructions at $\mathrm{s} 1 \mathrm{e} 3 \mathrm{r}, \mathrm{x} 0$ and $\mathrm{mrs} \mathrm{x} 0$, PAREL1 are written at a given address, then the $\mathrm{x} 0$ register is set to one virtual address, the two instructions are executed, and finally, the $\mathrm{x} 0$ register contains the corresponding physical address.

With this method, we compare the memory mappings with (Table 3 ) and without (Table 2) a fault.

\begin{tabular}{|c|c|c|c|}
\hline Virtual Address & $\begin{array}{l}\text { Physical } \\
\text { Address }\end{array}$ & Virtual Address & $\begin{array}{l}\text { Physical } \\
\text { Address }\end{array}$ \\
\hline $0 \times 0$ & $0 \times 0$ & $0 \times 80000$ & $0 \times 80000$ \\
\hline $0 \times 10000$ & $0 \times 10000$ & $0 \times 90000$ & $0 \times 90000$ \\
\hline $0 \times 20000$ & $0 \times 20000$ & $0 \times a 0000$ & $0 \times a 0000$ \\
\hline $0 \times 30000$ & $0 \times 30000$ & $0 x b 0000$ & $0 x b 0000$ \\
\hline $0 \times 40000$ & $0 \times 40000$ & $0 x c 0000$ & $0 \mathrm{xc} 0000$ \\
\hline $0 \times 50000$ & $0 \times 50000$ & $0 x d 0000$ & $0 x d 0000$ \\
\hline $0 \times 60000$ & $0 \times 60000$ & $0 x e 0000$ & $0 x e 0000$ \\
\hline $0 \times 70000$ & $0 \times 70000$ & $0 x f 0000$ & $0 \times f 0000$ \\
\hline
\end{tabular}

Table 2: Correct identity mapping

\begin{tabular}{|c|c|c|c|}
\hline Virtual Address & $\begin{array}{l}\text { Physical } \\
\text { Address }\end{array}$ & Virtual Address & $\begin{array}{l}\text { Physical } \\
\text { Address }\end{array}$ \\
\hline $0 \times 0$ & $0 \times 0$ & $0 \times 80000$ & $0 \times 0$ \\
\hline $0 \times 10000$ & $0 \times 10000$ & $0 x 90000$ & $0 \times 0$ \\
\hline $0 \times 20000$ & $0 \times 20000$ & $0 \times a 0000$ & $0 \times 0$ \\
\hline $0 \times 30000$ & $0 \times 30000$ & $0 x b 0000$ & $0 \times 0$ \\
\hline $0 \times 40000$ & $0 x 40000$ & $0 x c 0000$ & $0 x 80000$ \\
\hline $0 \times 50000$ & $0 \times 50000$ & $0 x d 0000$ & $0 \times 90000$ \\
\hline $0 \times 60000$ & $0 \times 60000$ & $0 x e 0000$ & $0 \times a 0000$ \\
\hline $0 \times 70000$ & $0 \times 70000$ & $0 x f 0000$ & $0 x b 0000$ \\
\hline
\end{tabular}

Table 3: Mapping after fault

Three different effects can be observed depending on the page:

1. Pages are correct with an identity mapping up to 0x70000. Remarkably, these are all the pages used to map our application in memory. Therefore, a hypothesis is that the corresponding translations are present in caches and are not impacted by the fault (in green in Table 3). 
2. Pages are incorrectly mapped to $0 x 0$. For instance, a read at 0x80000 reads, with success, physical memory at $0 \times 0$ (in blue in Table 3 ).

3. Pages are shifted. For example, a read at 0xc0000 gives the physical memory value at 0x80000 (in red in Table 3).

If we invalidate the TLB after a fault, nothing changes: the mapping is still modified. We conclude that the fault does not affect the cache mechanism to address translation (at least what can be invalidated by software) but directly the MMU configuration.

The fault on the MMU shifts the page tables in memory and inserts errors on it. The tables are still present, with small modifications, but at another base address.

Since the memory translation is still valid after the fault (translations are considered valid by the CPU), and do not correspond to the shifted page tables (we can reconstruct the virtual to physical mapping from the new page tables), this shift is not the only source of incorrect translation. Finally, we do not have enough control over the CPU to observe the failure cause with precision.

\subsection{Exploit}

This fault shows that the cornerstone of the key security feature in any CPU, namely memory isolation, does not withstand fault injection. In [23], the authors use the Rowhammer attack to fault a PTE. The faulted PTE accesses the kernel memory, which allows the attacker to obtain a privilege escalation: by overwriting a userland PTE for accessing all the memory, by changing the user ID to root or by changing the entry point of an executable.

Additionally, this fault model is a threat to pointer authentication countermeasures, as proposed in the recent ARMv8.3 ISA. This pointer protection mechanism works by storing authentication metadata in the most significant bits (usually useless) of a pointer value. The chip first validates the authentication metadata in order to use a pointer. In our case, the attacker does not need to alter the pointer value; it can alter where it physically points to, at a coarse (page) granularity.

\section{Shifting data chunks in L2}

We showed that the attacker could inject faults in the L1I cache and the MMU. In this section, we will demonstrate that another cache memory can be targeted: the L2 cache.
Another interesting behavior when faulting a modified version of the loop target, c.f. Listing 3, was investigated with JTAG.

The initial goal was to try to modify register values to see if we could confirm the fault model as in [21]. Instead, we observed an original behavior described in this section. Inside each loop iteration, we call a function, register_transfer, that self copies all registers (e.g., mov $\mathrm{x} 1, \mathrm{x} 1)$. init_registers fills registers $\mathrm{x} 0$ to $x 7$ with constants (9 instructions with the ret).

Listing 3: Assembly for the modified loop target, the framed instructions have been added with respect to Listing 2. We also show the instructions just before in our function.

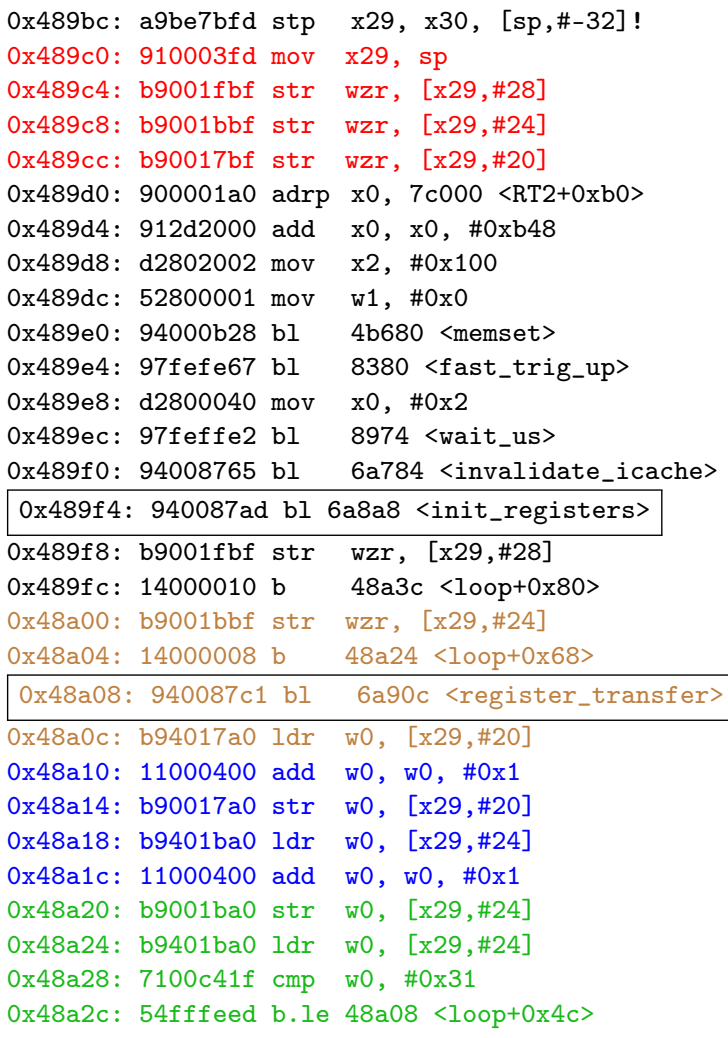

The pulse parameters are strictly identical to the ones used for injecting a fault on L1I (in particular, we have the same delay of $3144 \mathrm{~ns}$ ). But the different observed behaviors may be explained by the modification of the program under test (framed instructions in Listing 3 ). This modification has, in our opinion, no effect on the fault due to the functionality, but instead because it modifies the cache timings.

We show in this section that data are shifted in the L2 cache as if addresses had been slightly modified in the transfer from RAM to L2. 


\subsection{Forensic}

\subsubsection{Cache coherency methodology}

The difficulty to identify the presence of a fault in the cache L2 is that we cannot observe it. Instead, we will locate the fault indirectly by testing the coherency of the L1I, L1D and L2 caches with the methods illustrated in Figures 4, 5 and 6 .

First, to check the coherency of L1D and L1I, we use the JTAG as illustrated on Figure 4. We compare the instructions read from L1D with the JTAG with the ones executed (fetched from L1I) by checking on their side effects, one instruction at a time.

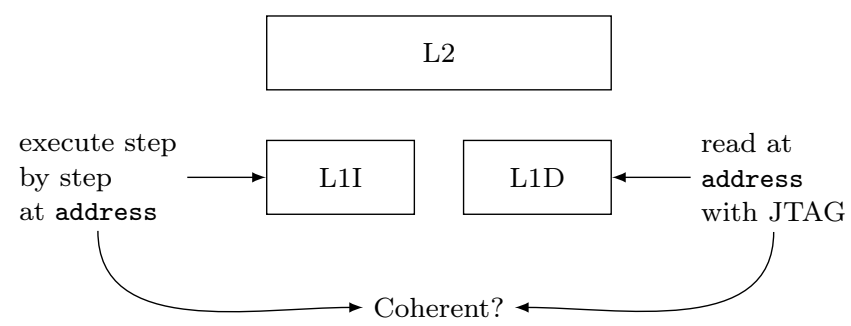

Fig. 4: Method for testing cache coherency between L1D and L1I using JTAG

Next, to verify the coherency of L1D with respect to L2, we force a new fetch from L2 after invalidating L1D as illustrated on Figure 5.

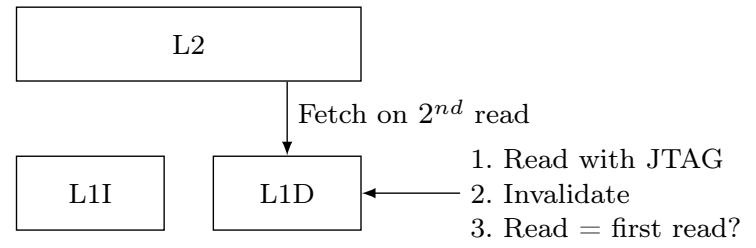

Fig. 5: Method for testing cache coherency between L1D and L2 using JTAG

Finally, it is also possible to force a new fetch from L2 after invalidating L1I ( $c f$. Figure 6). We compare the executions before and after this new fetch by executing instructions step-by-step.

\subsubsection{Incoherent cache fault}

With the same injection parameters as in section 5, we can achieve a new reproducible faulted behavior where the chip is trapped in an infinite loop, as seen with a step-by-step execution. However, when we repeat

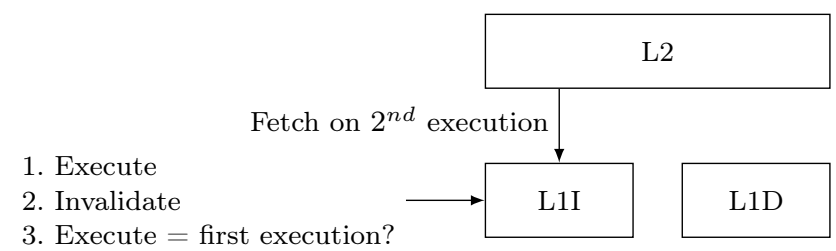

Fig. 6: Method for testing cache coherency between L1I and L2 using JTAG

the fault and observe the dumps with JTAG, we have two observable states (both are reproducible), called CoherentState and IncoherentState, that differ by the data read in L1D.

A JTAG memory dump (from L1D point of view) at the instruction memory location for CoherentState shows modified instructions as seen on Listing 4a, to be compared to the unfaulted dump on Listing $4 \mathrm{~b}$.

The dump for IncoherentState shows different values in L1D memory with respect to CoherentState (for the first two instructions), as seen on the Listing 5 .

Listing 5: Memory dump showing the instructions in the infinite loop as seen by the JTAG (L1D point of view) for IncoherentState.

0x000489f8: 940087c1 b94017a0 11000400 b90017a0

0x00048a08: b9401ba0 11000400 b9001ba0 b9401ba0

0x00048a18: 7100c41f 54fffeed b9401fa0 11000400

0x00048a28: b9001fa0 b9401fa0 7100c41f 54fffded

The step-by-step execution is coherent with the instructions shown by the JTAG dump for CoherentState (registers are loaded, stored and incremented as specified in this dump), which means that L1I and L1D are identically faulted. Therefore, we can suppose that the fault is present in the L2 cache (the first unified cache). The reconstructed instructions can be seen in Listing 6 .

Listing 6: Assembly reconstruction of the faulted instructions from the Listing $4 \mathrm{a}$.

\begin{tabular}{lll}
\hline address & binary value & \multicolumn{2}{c}{ Instruction } \\
0x489f8: & b9001bbf & str wzr, [x29,\#24] \\
0x489fc: & b90017bf & str wzr, [x29,\#20] \\
0x48a00: & 11000400 & add w0, w0, \#0x1 \\
0x48a04: & b90017a0 & str w0, [x29,\#20] \\
0x48a08: & b9401ba0 & ldr w0, [x29,\#24] \\
0x48a0c: & 11000400 & add w0, w0, \#0x1 \\
0x48a10: & b9001ba0 & str w0, [x29,\#24] \\
0x48a14: & b9401ba0 & ldr w0, [x29,\#24] \\
0x48a18: & $7100 c 41 f$ & cmp w0, \#0x31 \\
0x48a1c: & $54 f f f e e d$ & b.le 489f8 \\
\hline
\end{tabular}


0x489d8: d2800040 97feffe2 0000000200000008 0x489e8: $0000000200000008910003 f d$ b9001fbf 0x489f8: b9001bbf b90017bf 11000400 b90017a0 0x48a08: b9401ba0 11000400 b9001ba0 b9401ba0 0x48a18: 7100c41f 54fffeed b9401fa0 11000400 0x48a28: b9001fa0 b9401fa0 8104081477777777

(a) Memory dump showing the instructions as seen by the JTAG (L1D point of view) for CoherentState. The instructions in the infinite loop are underlined. 0x489b8: d65f03c0 a9be7bfd 910003fd b9001fbf 0x489c8: b9001bbf b90017bf 900001a0 912d2000 0x489d8: d2802002 $5280000194000 b 2897 f$ efe 67 0x489e8: d2800040 97feffe2 94008765 940087ad 0x489f8: b9001fbf 14000010 b9001bbf 14000008 0x48a08: 940087c1 b94017a0 11000400 b90017a0 0x48a18: b9401ba0 11000400 b9001ba0 b9401ba0 0x48a28: 7100c41f 54fffeed b9401fa0 11000400

(b) Memory dump showing correct (without fault) instructions for the same memory region.

Listing 4: Comparison of the memory dumps with and without fault.

It is impossible to observe the L2 cache content, we must therefore confirm our hypothesis with additional indirect evidence, using our methodology introduced in section 6.1.1.

L1 fault. The L1I is modified by the fault since the behavior of the application is modified. However, the execution is coherent with the JTAG dump (showing the L1D cache) for CoherentState, hinting that L2 is impacted too.

In this case, if we invalidate the whole L1I cache (with ic iallu instruction), as illustrated on Figure 6, nothing changes (either in the execution trace or in the JTAG dump).

For IncoherentState, 940087c1 (first brown instruction in Listing 5) encodes a direct unconditional jump (bl) instruction that is not followed in the stepby-step execution. Therefore, in this case the JTAG dump (corresponding to data in L1D) does not reflect the values in the L1I cache. However, if we invalidate a cache line of L1D at address $0 \times 489 \mathrm{f} 8$ (with dc ivac instruction), as illustrated on Figure 5, then L1D is modified, as seen with JTAG, and becomes the same as for CoherentState. Therefore, the L1D data seen in CoherentState is present in L2.

However, to the best of our knowledge, it is not possible to directly invalidate the L2 cache to directly confirm our hypothesis.

memory management unit. A possibility that would explain coherent faults in L1D and L1I but not L2 is a modification of the memory mapping if the fault impacted the MMU.

After verification as in section 5, we observe that the MMU mapping is indeed modified but not for the memory region of interest. In particular, memory addresses from $0 x 0$ to $0 x 7 F F F F$ are still correctly mapping identically virtual and physical addresses. The fault on the MMU cannot, therefore, explain our observations.
Hypotheses. The effect of these faults seems to manifest itself in the L2 cache. We can observe that it consists in shifting groups of 4 instructions (128 bits or $16 \mathrm{~B}$, under the cache line size of $64 \mathrm{~B}$ ) at a nearby memory location. 128-bit is the size of the external memory bus connected to L2. The fault model could be that the address corresponding to a cache transfer toward L2 has been modified by a few bits.

Our model has some limits: with one fault, values in L2 and in L1D are modified simultaneously in an incoherent way for IncoherentState. Is it due to the EM probe coupling with several buses or to a microarchitectural mechanism?

\subsection{Exploits}

A fault in the L2 cache can impact either the instructions or the data giving more power to the attacker. Nevertheless, she does not control in what way the memory will be modified. Why have these particular 16byte blocks been shifted? Nevertheless, just corrupting data or instruction randomly is often enough to achieve the desired effect (as in the NOP fault model).

\section{When persistent fault analysis meets L1D cache}

To demonstrate the applicability of a cache memory fault model, a persistent fault analysis (PFA) [29] is performed on a bare-metal AES implementation. In this section, we show how to reduce the AES key to $2^{24}$ possibilities instead of $2^{128}$, with one persistent fault in L1D.

\subsection{Setup}

With the same experimental setup as before, we now target an AES encryption. Our AES implementation is 
a naive one without countermeasures. The sboxes are implemented with a lookup table. Before the actual encryption, we pre-warm the cipher: in order to limit timing leakages due to caches, we first invalidate L1I and L1D. Then we perform a dummy AES encryption to fill the caches with data and instructions of interest. This is the step where we inject the fault.

In a second step, we encrypt 10000 random plaintexts, without any new fault injection, and try to deduce the key from the observed (faulty) ciphertexts.

\subsection{Persistent fault analysis: the theory}

We target the sbox lookup table: by modifying at least one bit in the table, the operation is now biased. For example, for the input $x$, the output is now

$y^{*}=S B^{*}(x)$

instead of $y=S B(x)$.

Since a sbox is a bijection, now $y$ cannot be outputted by $S B^{*}$, the faulted sbox. We say that $y$ is forbidden. At the last round of the AES encryption, for each byte index $i \in \llbracket 0,15 \rrbracket$, we have

$S B^{*}\left(x_{i}\right) \oplus K 10_{i}=C_{i}^{*}$,

where $x$ is an intermediate state, $K 10$ the last round key and $C^{*}$ the faulty ciphertext. We consider, as an approximation, that the distribution of $x_{i}$ is uniform for all $i$.

Since $y$ is a forbidden value, $y \oplus K 10_{i}$ cannot be observed as a result for any $C_{i}^{*}$ and this for all byte index $i \in \llbracket 0,15 \rrbracket$. We note $C_{i}^{0}$ the forbidden value for the corresponding ciphertext at byte index $i$. The attacker can easily observe $C_{i}^{0}$, it is enough to count the occurrences of the byte values and find the specific one that never occurs.

While we try to guess for the forbidden value $y\left(2^{8}\right.$ possibilities), we can compute:

$\forall i, K 10_{i}=C_{i}^{0} \oplus y$

From there, the master key is trivially recovered from $K 10$, and a known pair with a plaintext and the corresponding correct ciphertext allows the attacker to validate the correct guess.

\subsection{Results}

In order to detect the forbidden values, we simply count the number of occurrences for all bytes values in the ciphertext, for the first byte, then the second byte, etc.
On Figure 7, we can observe that 2-byte values are forbidden. Therefore, in our case, two bytes have been faulted in the sbox lookup table.

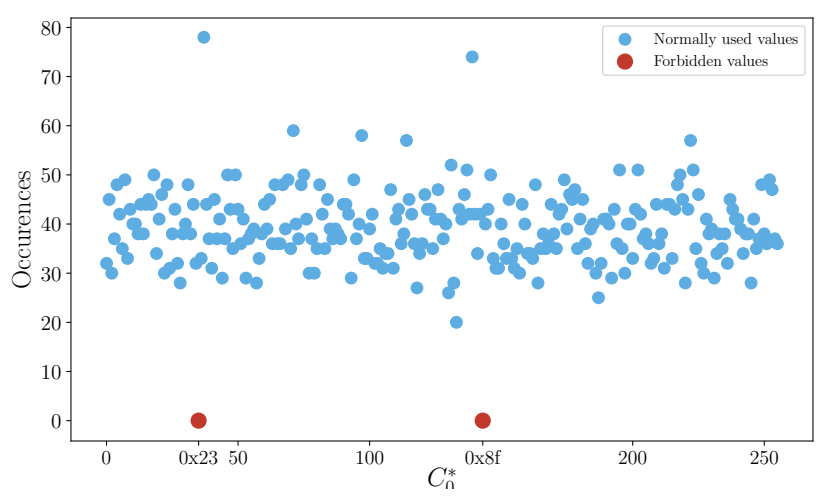

Fig. 7: For the first ciphertext byte, a number of occurrences for each value; for a total of 10000 occurrences.

The analysis has to be adapted to the two faults case. For each byte index from 0 to 15 , we get the two ciphertext forbidden values, as shown in Table 4.

\begin{tabular}{|l|c|c|c|c|c|c|c|c|}
\hline Index & 0 & 1 & 2 & 3 & 4 & 5 & 6 & 7 \\
\hline Forbidden & 23 & 21 & $2 \mathrm{~d}$ & $4 \mathrm{f}$ & $7 \mathrm{f}$ & 08 & $7 \mathrm{a}$ & 27 \\
values & $8 \mathrm{f}$ & $8 \mathrm{~d}$ & 81 & $\mathrm{e} 3$ & $\mathrm{~d} 3$ & $\mathrm{a} 4$ & $\mathrm{~d} 6$ & $8 \mathrm{~b}$ \\
\hline \hline Index & 8 & 9 & 10 & 11 & 12 & 13 & 14 & 15 \\
\hline Forbidden & $6 \mathrm{f}$ & 37 & $3 \mathrm{~b}$ & 17 & $7 \mathrm{~d}$ & $1 \mathrm{~b}$ & 00 & 59 \\
values & $\mathrm{c} 3$ & $9 \mathrm{~b}$ & 97 & $\mathrm{bb}$ & $\mathrm{d} 1$ & $\mathrm{~b} 7$ & $\mathrm{ac}$ & $\mathrm{f5}$ \\
\hline
\end{tabular}

Table 4: Ciphertext forbidden values. For each byte index, we have 2 forbidden values. The first two forbidden values are the ones that we can observe in Figure 7.

Since we have 2 forbidden values $y_{1}$ and $y_{2}$, we need to create $2^{24}$ hypotheses to recover the key. First, we have $2^{8}$ guesses for $y_{1}$ then, for each guess, we need to choose one value out of two possibilities for each of the byte index ( $2^{16}$ possible choices). The possible key bytes for a correctly guessed forbidden value $y_{1}=30$ are displayed in Table 5. In other words, Table 5 is Table 4 xored with $y_{1}$. Finding the key then consists in choosing the correct value (shown in red on Table 5) out of the two, for each byte index.

In total, we have $2^{24}$ key hypotheses, with a pair of plaintext and corresponding ciphertext to validate the correct key, recovering the AES key can be done on a desktop computer in a few minutes. It takes $145 \mathrm{~s}$ on an Intel E5-1620 v3 CPU to find $y_{1}=30$, so it would take by extrapolation $757 \mathrm{~s}$, or less than fifteen minutes, to test all keys. 


\begin{tabular}{|l|c|c|c|c|c|c|c|c|}
\hline Index & 0 & 1 & 2 & 3 & 4 & 5 & 6 & 7 \\
\hline Forbidden & 13 & 11 & $1 \mathrm{~d}$ & $7 \mathrm{f}$ & $4 \mathrm{f}$ & 38 & $4 \mathrm{a}$ & $\begin{array}{c}17 \\
\text { values }\end{array}$ \\
$\mathrm{bf}$ & $\mathrm{bd}$ & $\mathrm{b} 1$ & $\mathrm{~d} 3$ & $\mathrm{e} 3$ & 94 & $\mathrm{e} 6$ & $\mathrm{bb}$ \\
\hline \hline Index & 8 & 9 & 10 & 11 & 12 & 13 & 14 & 15 \\
\hline Forbidden & $5 \mathrm{f}$ & 07 & ob & 27 & $4 \mathrm{~d}$ & $2 \mathrm{~b}$ & 30 & 69 \\
values & $\mathrm{f} 3$ & $\mathrm{ab}$ & $\mathrm{a} 7$ & $8 \mathrm{~b}$ & $\mathrm{e} 1$ & 87 & $9 \mathrm{c}$ & $\mathrm{c5}$ \\
\hline
\end{tabular}

Table 5: Possible key bytes for the correct guess $y_{1}=$ 30. The correct $K 10$ key is 1311 dd $7 \mathrm{f}$ e3 $94 \quad 4$ a 17 f3 07 a7 8b 4d 2b 30 c5.

\subsection{Forensic}

The fault exploited here is actually in L1D and can be directly observed with the JTAG. We show on Listings 7 and 8 the correct and faulty lookup tables as seen by the JTAG.

0x0006b510: 7b777c63 c56f6bf2 2b670130 76abd7fe 0x0006b520: 7dc982ca f04759fa afa2d4ad c072a49c

Listing 7: Part of the correct sbox lookup table.

0x0006b510: 7b777c63 c56f6bf2 2b670136 76abd7fe 0x0006b520: 7dc982ca f04759fa afa2d4ad c072a498

Listing 8: Part of the faulty sbox lookup table. The two faults are displayed in red.

We can notice that the sbox output 30 has been replaced by 36.30 is, therefore, a forbidden value at sbox output, at the origin of the correct guess $y_{1}=30$.

The two faulted values are more than 128 bits apart, but on the same cache line (if we suppose that they are aligned in memory). The only memory bus ( $c f$. Table 1$)$ with a size greater than 128 bits is the one that write L2 from L1D values. Since we are reading sbox values, not writing them, it is highly improbable that such a bus transfer was hit. Instead, we should consider that the fault impacted several memory transfers, which has been shown possible in section 6 by the incoherence between the L2 and L1D caches in the IncoherentState case.

\section{Defense mechanisms}

In this paper, we have shown that you cannot rely on hardware for critical functionality. Our forensic analyses show that the memory hierarchy and the MMU can easily be corrupted. However, other micro-architectural elements might also be confused by the injected fault.
We can conjecture that the subsystems ensuring data coherence, speculative execution, etc. will one day be the victims of fault attacks.

As such, protecting a CPU against fault attacks requires to ensure the integrity of their micro-architectural blocks. If this problem is well known for secure components such as smartcards, hardening blocks which compose a modern and complex CPU is an open problem.

In this section, we discuss how to protect complex CPUs to fault injection attacks inside the CPU or out of it through the SoC organization.

\subsection{Inside the $\mathrm{CPU}$}

Instead of solving the integrity problem for all microarchitectural blocks, which is a complex research program in itself, we focus on the cache memories.

\subsubsection{Ensuring integrity and authenticity in cache memories}

Two strategies can be deployed to detect memory faults. On the one hand, a common solution to quickly verify data integrity in memory hierarchy is the error correcting codes (ECCs). They are efficient to detect a a limited number random faults : this solution is relatively low-cost but assumes that the attacker has no control over the fault value and cannot inject a lot of faults. In the other case, the attacker would be able to inject random faults until one passes the ECC scheme.

If we only consider the attacks introduced in this paper, this mechanism is enough due to the lack of control on the fault injection and the low automation of the forensic process; the fault rate is low if we need to check the fault effects. However, face a more powerful attacker, it can only slightly slow the attack down.

On the other hand, the use of cryptography in the form of one message authentication code (MAC) can detect memory faults. A generic solution is KECCAK message authentication code (KMAC) with a small output length since the lifetime of the integrity and authenticity protection is limited and to reduce the memory overhead. With an output length of 64 bits (we store $8 \mathrm{~B}$ of MAC for each $64 \mathrm{~B}$ cache line), the protection increases by $12.5 \%$ the memory footprint than the unprotected version (without taking into account tags and flags).

Key generation. The key $k$ is a true random value that the system should generate at boot time. It must be kept secret so that the attacker cannot know the correct $\mathrm{MAC}$ value for a given cache line. 
Signing. For each cache line modification, total or partial, the KMAC must be computed again. For a cache line tag $t$, data block $d$ and flags $f$ let $h=K M A C(k, t|d| f)$ be the MAC value stored along the cache line. Instead of the tuple $(t, d, f)$, we now store $(t, d, f, h)$.

Verifying. When a cache line is read, the MAC must be verified. For a read in the cache line constituted by the tuple $(t, d, f, h)$, we compute $h_{\text {verif }}=K M A C(k, t|d| f)$. Finally, we compare $h$ and $h_{\text {verif }}$. If they are equal, the read value is correct. Otherwise, we trigger the alarm: an exception is raised to allow custom software management.

\subsubsection{Speculative memory accesses}

To ensure data integrity, a MAC is necessary against powerful attackers. However, computing a KMAC requires multiple cycles which drastically decrease the performances of the whole system, especially in the memory hierarchy which already constitutes a bottleneck.

To tackle this problem, we introduce a speculative mechanism for integrity verification. By default, all data coming from memory are considered as usable but not verified: we speculate that they are correct. Therefore, load instructions must be dealt with similarly to speculative branches: the core can continue its execution, but without committing the load until the integrity verification. If the chip is under attack, the verification would fail and the chip can be restored to its initial state, before the incriminated load.

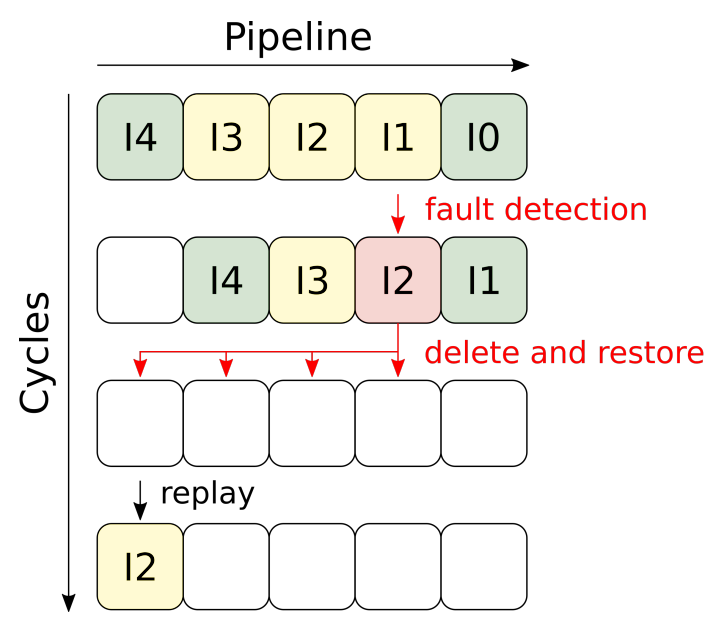

Fig. 8: Integrity speculation in a pipelined core. A green instruction means that no speculation is involved, a yellow one that speculation is pending. The red instruction denotes a speculative load that is dismissed.
Figure 8 illustrates with an example how integrity speculation can be managed in a pipelined core.

With this speculation strategy, verification and normal computation can be performed simultaneously. It allows increasing the latency margin to perform the verification, enabling the use of MACs. Obviously, this kind of mechanism would be made simpler and less critical by low latency cryptographic hashes, which have yet to be invented.

\subsubsection{The path to secure cores}

Complex cores today are mainly split into two classes, the high-performance ones and the low energy consumption ones. The security problems are addressed through software modification (e.g. retpoline [22] against the Spectre attack [11]) or minimal hardware modification (e.g. TrustZone to protect against rich OS vulnerabilities). Security is considered as an implementation issue, where attacks happen only if a designer made a mistake.

However, this work shows that a modern complex core is not a reliable substrate for secure computations. Generic secure cores must be designed to handle all the security needs. These secure cores will have to be designed from the ground up, to guarantee security properties across all micro-architectural blocks. We envision a future where the SoCs will be composed of 3 kinds of cores: a core can either grant high performance, low energy consumption or high security.

\subsection{Through the SoC organization}

It is worth mentioning a natural countermeasure that may complicate fault attacks on SoCs, 3D packaging. This technique consists in stacking the dies (CPU, memory, baseband, etc.) in the same package. Experimentally, the attacker may find it difficult to target dies that are not on the surface of the package. Yet it has been demonstrated possible: for example, Vasselle et al. [25] show a laser fault injection attack on a SoC where the external RAM is stacked on it.

\section{Related works}

In the literature, fault attacks are a hot topic where studies are focused on effects, targetting both MCUs and complex CPUs. Despite the works made on MCUs, recent complex CPUs are still vulnerable to fault attacks mainly because the manufacturers prefer performance or low consumption than security $[11,12]$. In this section, we made an overview of state-of-the-art fault 
attacks.

Fault attacks on hardware components have been studied starting from the seminal work of Skorobogatov et al. [17] in 2002. Significant works which focus on MCUs have been devoted to demonstrating how fault attacks can break security mechanisms where both cryptography [16, 24, 27] and critical applications [3, 5] are targeted.

Until recently, MCUs were the favourite targets since they are cheaper and simpler (no memory virtualization, simple cache levels if any, no speculative nor outof-order execution) than the complex CPUs. Therefore, it was a good target to evaluate the fault effects. Mainly within a black box approach, several works $[1,2,10$, $13,15,26]$ focus on characterizing fault effects from the ISA level. Characterizing ISA faults is an interesting approach when you have no access to the hardware IP. Another approach [4, 9] is to focus on characterizing faults at the level of transistors and logic gates.

Recently, inspired by the works made on MCUs, fault attacks targeting complex CPUs have emerged. They target the secure boot $[8,20,25]$, the trusted execution environment (TEE) [18], the memory partitioning [23] or some OS security mechanisms [19]. However, due to the complexity of both hardware and software on these targets, characterizing the fault effects are more difficult than on MCUs.

Characterizing fault effects on complex CPUs is a hot topic to improve their hardware security; for instance, to prevent attacks by implementing efficient countermeasures. Few works $[14,21]$ focus on this research area.

In [14], the authors develop a fault model at the ISA level. In other words, they identify how the data and the instructions of a program are affected by the fault. It is essential to understand the impact on a program and to propose software-based countermeasures, but some works have shown that software-based countermeasures are not sufficient [27] and hardware ones are needed. However, for building hardware countermeasures, the fault model must be characterized at the micro-architectural level, not the ISA level.

To complete this approach, Trouchkine et al. [21] propose a model for complex CPUs and a method to obtain micro-architectural information on the fault from observations at the ISA level. By using this method, the authors are able to identify the defects that appear in the registers, pipeline or cache of an x86 CPU and an ARM CPU. Although this method is able to identify the affected CPU micro-architectural components, due to the complex OS presence and from an ISA perspective, they are not able to accurately determine the micro-architectural fault model.

In our article, we focus on a target without a complex OS. Therefore, we relax hypotheses about the effect of a complex software on the fault effect characterization. Within this approach, we disclose new fault effects on the memory hierarchy.

\section{Conclusion}

In this paper, we have demonstrated that complex CPUs are vulnerable to fault injection attacks. In particular, the memory hierarchy and the MMU can be changed, which creates a mismatch between hardware behavior and software expectations. Nowadays, the security of computing systems is focused on the software side; there are no efficient countermeasures against hardware perturbations in complex CPUs. Pointer authentication, as proposed by the ARMv8.3 ISA, for example, does not resist the introduced fault model.

Exploitation heavily depends on the interaction between the hardware (the specific device) and the software (including applications and OS). Therefore the cautious developer cannot predict where vulnerabilities will occur and, as a consequence, cannot efficiently protect its application. Today, such attacks using EMFI are still quite hard to realize: they require expensive apparatus, human resources to do the experiments, etc. However, they are within reach of small organizations, and we can expect that the difficulty and cost of these attacks will be lower in the future.

Actions must be taken to ensure that computing systems handle sensitive information securely. The performances/energy consumption trade-off has been settled by implementing the two kinds of cores in the same SoC. In the same way, we need secure cores as well that compromise both on performances and energy consumption but can offer much stronger security guarantees.

\section{References}

1. J. Balasch, B. Gierlichs, and I. Verbauwhede. An in-depth and black-box characterization of the effects of clock glitches on 8-bit mcus. In 2011 Workshop on Fault Diagnosis and Tolerance in Cryptography, FDTC 2011, Tokyo, Japan, September 29, 2011, pages 105-114, 2011.

2. H. Bar-El, H. Choukri, D. Naccache, M. Tunstall, and C. Whelan. The sorcerer's apprentice guide to fault attacks. Proc. IEEE, 94(2):370-382, 2006. 
3. G. Barbu, H. Thiebeauld, and V. Guerin. Attacks on java card 3.0 combining fault and logical attacks. In D. Gollmann, J. Lanet, and J. IguchiCartigny, editors, Smart Card Research and Advanced Application, 9th IFIP WG 8.8/11.2 International Conference, CARDIS 2010, Passau, Germany, April 14-16, 2010. Proceedings, volume 6035 of Lecture Notes in Computer Science, pages 148163. Springer, 2010.

4. N. Beringuier-Boher, M. Lacruche, D. El-Baze, J. Dutertre, J. Rigaud, and P. Maurine. Body biasing injection attacks in practice. In M. Palkovic, G. Agosta, A. Barenghi, I. Koren, and G. Pelosi, editors, Proceedings of the Third Workshop on Cryptography and Security in Computing Systems, CS2@HiPEAC, Prague, Czech Republic, January 20, 2016, pages 49-54. ACM, 2016.

5. G. Bouffard, J. Iguchi-Cartigny, and J. Lanet. Combined software and hardware attacks on the java card control flow. In E. Prouff, editor, Smart Card Research and Advanced Applications 10th IFIP WG 8.8/11.2 International Conference, CARDIS 2011, Leuven, Belgium, September 14-16, 2011, Revised Selected Papers, volume 7079 of Lecture Notes in Computer Science, pages 283-296. Springer, 2011.

6. L. Breveglieri, S. Guilley, I. Koren, D. Naccache, and J. Takahashi, editors. 2011 Workshop on Fault Diagnosis and Tolerance in Cryptography, FDTC 2011, Tokyo, Japan, September 29, 2011. IEEE Computer Society, 2011.

7. S. K. Bukasa, R. Lashermes, J.-L. Lanet, and A. Legay. Let's shock our iot's heart: Armv7-m under (fault) attacks. In Proceedings of the 13th International Conference on Availability, Reliability and Security, ARES 2018, pages 33:1-33:6, New York, NY, USA, 2018. ACM.

8. A. Cui and R. Housley. BADFET: Defeating Modern Secure Boot Using Second-Order Pulsed Electromagnetic Fault Injection. In W. Enck and C. Mulliner, editors, 11th USENIX Workshop on Offensive Technologies, WOOT 2017, Vancouver, BC, Canada, August 14-15, 2017. USENIX Association, 2017.

9. M. Dumont, M. Lisart, and P. Maurine. Electromagnetic fault injection : How faults occur. In 2019 Workshop on Fault Diagnosis and Tolerance in Cryptography, FDTC 2019, Atlanta, GA, USA, August 24, 2019, pages 9-16. IEEE, 2019.

10. L. Dureuil, M. Potet, P. de Choudens, C. Dumas, and J. Clédière. From code review to fault injection attacks: Filling the gap using fault model inference. In N. Homma and M. Medwed, editors, Smart Card
Research and Advanced Applications - 14th International Conference, CARDIS 2015, Bochum, Germany, November 4-6, 2015. Revised Selected Papers, volume 9514 of Lecture Notes in Computer Science, pages 107-124. Springer, 2015.

11. P. Kocher, J. Horn, A. Fogh, D. Genkin, D. Gruss, W. Haas, M. Hamburg, M. Lipp, S. Mangard, T. Prescher, et al. Spectre attacks: Exploiting speculative execution. In 2019 IEEE Symposium on Security and Privacy (SP), pages 1-19. IEEE, 2019.

12. M. Lipp, M. Schwarz, D. Gruss, T. Prescher, W. Haas, A. Fogh, J. Horn, S. Mangard, P. Kocher, D. Genkin, Y. Yarom, and M. Hamburg. Meltdown: Reading kernel memory from user space. In W. Enck and A. P. Felt, editors, 27th USENIX Security Symposium, USENIX Security 2018, Baltimore, MD, USA, August 15-17, 2018, pages 973 990. USENIX Association, 2018.

13. N. Moro, A. Dehbaoui, K. Heydemann, B. Robisson, and E. Encrenaz. Electromagnetic fault injection: Towards a fault model on a 32-bit microcontroller. In 2013 Workshop on Fault Diagnosis and Tolerance in Cryptography, Los Alamitos, CA, USA, August 20, 2013, pages 77-88, 2013.

14. J. Proy, K. Heydemann, F. Majéric, A. Berzati, and A. Cohen. A first isa-level characterization of em pulse effects on superscalar microarchitectures - a secure software perspective. In ARES 2019, 2019.

15. L. Rivière, Z. Najm, P. Rauzy, J. Danger, J. Bringer, and L. Sauvage. High precision fault injections on the instruction cache of armv7-m architectures. In IEEE International Symposium on Hardware Oriented Security and Trust, HOST 2015, Washington, DC, USA, 5-7 May, 2015, pages 62-67, 2015.

16. J. Schmidt and C. Herbst. A practical fault attack on square and multiply. In L. Breveglieri, S. Gueron, I. Koren, D. Naccache, and J. Seifert, editors, Fifth International Workshop on Fault Diagnosis and Tolerance in Cryptography, 2008, FDTC 2008, Washington, DC, USA, 10 August 2008, pages 53-58. IEEE Computer Society, 2008.

17. S. P. Skorobogatov and R. J. Anderson. Optical fault induction attacks. In B. S. K. Jr., Ç. K. Koç, and C. Paar, editors, Cryptographic Hardware and Embedded Systems - CHES 2002, 4th International Workshop, Redwood Shores, CA, USA, August 1315, 2002, Revised Papers, volume 2523 of Lecture Notes in Computer Science, pages 2-12. Springer, 2002.

18. A. Tang, S. Sethumadhavan, and S. J. Stolfo. CLKSCREW: exposing the perils of securityoblivious energy management. In E. Kirda and 
T. Ristenpart, editors, 26th USENIX Security Symposium, USENIX Security 2017, Vancouver, BC, Canada, August 16-18, 201\%., pages 1057-1074. USENIX Association, 2017.

19. N. Timmers and C. Mune. Escalating privileges in linux using voltage fault injection. In 2017 Workshop on Fault Diagnosis and Tolerance in Cryptography, FDTC 2017, Taipei, Taiwan, September 25, 2017, pages 1-8. IEEE Computer Society, 2017.

20. N. Timmers, A. Spruyt, and M. Witteman. Controlling $\mathrm{PC}$ on ARM using fault injection. In 2016 Workshop on Fault Diagnosis and Tolerance in Cryptography, FDTC 2016, Santa Barbara, CA, USA, August 16, 2016, pages 25-35, 2016.

21. T. Trouchkine, G. Bouffard, and J. Clediere. Fault Injection Characterization on modern CPUs From the ISA to the Micro-Architecture. In WISTP 2019, Paris, France, 2019.

22. P. Turner. Retpoline: a software construct for preventing branch-target-injection. https:// support. google. com/faqs/answer/7625886, 2018.

23. V. van der Veen, Y. Fratantonio, M. Lindorfer, D. Gruss, C. Maurice, G. Vigna, H. Bos, K. Razavi, and C. Giuffrida. Drammer: Deterministic rowhammer attacks on mobile platforms. In Proceedings of the 2016 ACM SIGSAC Conference on Computer and Communications Security, Vienna, Austria, October 24-28, 2016, pages 1675-1689, 2016.

24. J. G. J. van Woudenberg, M. F. Witteman, and F. Menarini. Practical optical fault injection on secure microcontrollers. In Breveglieri et al. [6], pages $91-99$.

25. A. Vasselle, H. Thiebeauld, Q. Maouhoub, A. Morisset, and S. Ermeneux. Laser-induced fault injection on smartphone bypassing the secure boot. In 2017 Workshop on Fault Diagnosis and Tolerance in Cryptography, FDTC 2017, Taipei, Taiwan, September 25, 2017, pages 41-48, 2017.

26. I. Verbauwhede, D. Karaklajic, and J. Schmidt. The fault attack jungle - A classification model to guide you. In Breveglieri et al. [6], pages 3-8.

27. B. Yuce, N. F. Ghalaty, H. Santapuri, C. Deshpande, C. Patrick, and P. Schaumont. Software fault resistance is futile: Effective single-glitch attacks. In 2016 Workshop on Fault Diagnosis and Tolerance in Cryptography, FDTC 2016, Santa Barbara, CA, USA, August 16, 2016, pages 47-58. IEEE Computer Society, 2016.

28. B. Yuce, P. Schaumont, and M. Witteman. Fault attacks on secure embedded software: Threats, design, and evaluation. Journal of Hardware and Systems Security, 2(2):111-130, Jun 2018.
29. F. Zhang, X. Lou, X. Zhao, S. Bhasin, W. He, R. Ding, S. Qureshi, and K. Ren. Persistent fault analysis on block ciphers. IACR Transactions on Cryptographic Hardware and Embedded Systems, 2018(3):150-172, Aug. 2018. 